\title{
TOPOGRAPHIC LOCAL ROUGHNESS EXTRACTION AND CALIBRATION OVER MARTIAN SURFACE BY VERY HIGH RESOLUTION STEREO ANALYSIS AND MULTI SENSOR DATA FUSION
}

\author{
J. R. Kim ${ }^{\mathrm{a}, *}$, J. G. Park ${ }^{\mathrm{b}}$ \\ ${ }^{\text {a }}$ Department of Geoinformatics, University of Seoul, Seoul - Korea - 130-875-kjrr001@gmail.com \\ ${ }^{\mathrm{b}}$ Korea Advanced Institute of Science and Technology, Daejoon - Korea - 130-875 - pjiga06@kaist.ac.kr
}

WG VI/7

KEY WORDS: Stereoscopic, planetary, Extraterrestrial, Surface, Three dimensional, Extraction

ABSTRACT:

The planetary topography has been the main focus of the in-orbital remote sensing. In spite of the recent development in active and passive sensing technologies to reconstruct three dimensional planetary topography, the resolution limit of range measurement is theoretically and practically obvious. Therefore, the extraction of inner topographical height variation within a measurement spot is very challengeable and beneficial topic for the many application fields such as the identification of landform, Aeolian process analysis and the risk assessment of planetary lander. In this study we tried to extract the topographic height variation over martian surface so called local roughness with different approaches. One method is the employment of laser beam broadening effect and the other is the multi angle optical imaging. Especially, in both cases, the precise pre processing employing high accuracy DTM (Digital Terrain Model) were introduced to minimise the possible errors. Since a processing routine to extract very high resolution DTMs up to $0.5-4 \mathrm{~m}$ grid-spacing from HiRISE (High Resolution Imaging Science Experiment) and 20-10m DTM from CTX (Context Camera) stereo pair has been developed, it is now possible to calibrate the local roughness compared with the calculated height variation from very high resolution topographic products. Three testing areas were chosen and processed to extract local roughness with the co-registered multi sensor data sets. Even though, the extracted local roughness products are still showing the strong correlation with the topographic slopes, we demonstrated the potentials of the height variations extraction and calibration methods.

\section{INTRODUCTION}

Nodaway, the spatial resolution and coverage of $3 \mathrm{D}$ planetary topography have been dramatically increased by a line of the successful exploration missions. Especially stereo analyses employing the very high resolution optical sensors such as HiRISE equipped on MRO (Mars Reconnaissance Orbiter) and Narrow Angle image of LRO (Lunar Reconnaissance Orbiter) are the important data sources for the 3D planetary topography construction. It provided not only the detailed $3 \mathrm{D}$ geomorphic information over planetary surface but also the height variation in a fine spatial resolution. Since the coverage of very high resolution stereo images over target planetary and satellite is usually very limited, the fine resolution height variation by the very high resolution stereo interpretation can be determined in only small part of planetary surface. Therefore, it should be highly valuable if the estimation of height variation within a unit measurement of remote sensing, so called the local roughness, is feasible.

The importance of local roughness in terrestrial and extra terrestrial geomorphic process has been noticed by many researchers. Especially the relationship with the aeolian process was explored even by very early research work (Bagnold, 1941) and recently was newly studied by Marticrena and Bergametti (1995). Since some plants, especially Mars, have potential condition and the evidences to make such aeolian surface process, it is highly desirable to extract the local roughness over wide surface coverage. On the other hands, the landing site selection of future planetary lander and rover is requiring the fine resolution height variation information to minimise the engineering risk as shown in Glomombeck et al., (2003). It is likely possible that the topographic textures from the different origins such as glacial, fluvial, aeolian and volcanic activities result in the different local roughness parameters. In that case, the local roughness distribution will give an important clue about the origin of planetary surface.

In this study we tested possible approaches to extract the local roughness by the multi sensor data fusions of medium resolution remote sensing information which is globally available in the planetary surface.
Two multi sensor data fusion approaches to extract local roughness were performed on martian surface where various remote sensing data are available. At first, the surface local roughness using the MOLA laser beam broadening effect (Gardner, 1992) combining the slope of HRSC (High Resolution Stereo Camera) and/or CTX stereo DTM were processed. The technical difficulties such as across track drifts of laser beam broadening have been tackled by introducing the adaptive filtering. Secondly, the local roughness index by the multi angle image interpretation after applying sub pixel co-registration between the corresponding pixels of different viewing channels were extracted. The data sets from these two approaches were verified by the directly calculated roughness parameters from very high resolution stereo height measurements and their correlations were investigated.

\section{BACKGROUND AND ALGORITHMS}

In the local roughness extraction, the first issue to be considered is the coverage of local roughness information source. For example, the stereo coverage of sub meter HiRISE optical image is only less than $1 \%$ of martian surface. Therefore, the direct extraction of height variation by the lateral cover measurement from 3D range data sources as shown in Marticorena et al. (2006) is extremely difficult and rarely possible for the purposes in all above topics. Hence, the circuitous approaches to extract the local roughness parameters from the medium resolution remote sensing data were employed. After then the calibration procedures were performed compared with the roughness parameters defined by Shepard et al., (2001) as described in below.

\subsection{MOLA beam broadening data processing}

LiDAR measurements over topography are influenced by the slope angles and the local roughness of the target area. In the simple description, the higher slope angle and roughness increased the duration of the returned laser beam.

Garder (1992) interrelated the influence of various topographic effects on the laser pulse width as follows

\footnotetext{
* Corresponding author
} 


$$
\begin{aligned}
& E\left(\sigma_{p^{2}}\right)=\left(\sigma_{l}^{2}+\sigma_{h}{ }^{2}\right)+\frac{4 \operatorname{Var}(\xi) \cos ^{2} S_{\mathrm{II}}}{c^{2} \cos ^{2}\left(\phi+S_{\mathrm{II}}\right)}+ \\
& \frac{4 R_{m}{ }^{2}}{c^{2}} \tan ^{2} \theta_{t}\left(\tan ^{2} \theta_{t}+\tan ^{2}\left(\phi+S_{I I}\right)+\frac{\tan ^{2} S_{\perp} \cos ^{2} S_{I I}}{\cos ^{2}\left(\phi+S_{I I}\right)}\right)
\end{aligned}
$$

where $\sigma_{I}$ is the rms laser pulse width, $\sigma_{h}$ is the rms width of the receiver impulse response, $R m$ is the range from the spacecraft to the illuminated spot, $S_{\mathrm{II}}$ is the surface slope parallel to nadir direction, $S_{\perp}$ is the surface slope to the nadir direction, $\vartheta_{t}$ is the nominal divergence angle, $c$ is the light speed and $\varphi$ is the off nadir angle. In equation (1), the first term represents a system effect, the second is the influence of height undulation and the third term is the effect of slope. Usually the off nadir angle is almost zero and the nominal divergence angle is relatively small, so (1) can be simplified as

$$
E\left(\sigma_{p^{2}}\right)=\left(\sigma_{l}{ }^{2}+\sigma_{h}{ }^{2}\right)+\frac{4 \operatorname{Var}(h)}{c^{2}}+\frac{4 R_{m}{ }^{2}}{c^{2}} \tan ^{2} \theta_{t}\left(\tan ^{2} S_{I I}+\tan ^{2} S_{\perp}\right)
$$

where $\operatorname{Var}(h)$ is the variance of height.

Therefore the local surface roughness can be derived by applying correction terms as follows

$$
\text { roughness }_{r m s}=0.5 c \sqrt{\sigma_{\text {corr }}{ }^{2}-\frac{4 R_{m}^{2}}{c^{2}} \tan ^{2} \theta_{t}\left(\tan ^{2} S_{I I}+\tan ^{2} S_{\perp}\right)} \text { (3) }
$$

Since MOLA (Mars Obiter Laser Altimeter) has enough coverage and the recording function of pulse width to apply above correction, it is possible to extract topographic roughness from MOLA beam broadening. In here, $\sigma_{\text {corr }}$ is the corrected pulse width which is recorded in the MOLA PEDR data where correction refers to the filter characteristics and threshold settings, as determined by the receiver model (one sigma value, with the minimum limited by the filter response; Neumannet al., 2003).

However the conversion procedure of $\sigma_{\text {corr }}$ into roughness value requires two pre-processing stages. First of all, the MOLA tracks frequently including the invalid noisy portion (Kim and Muller, 2008) have to be validated. Secondary the correction procedure of the slope effects in (3) using the topographic data set, usually MOLA $3 \mathrm{D}$ range values, is necessary.

However, in our experience even with all above corrections, quite significant artefacts especially crossing MOLA tracks are usually unresolved. The reason of such across track artefacts is that some vertical range errors within individual tracks were not corrected. Especially between tracks, there are some uncertainties in recorded MOLA pulse widths. Moreover, the big across track gaps $(\approx 4 \mathrm{~km})$ produced wider baseline for the across track slope calculation compared with the MOLA beam footprint size $(<120 \mathrm{~m})$. The multi beam observation mechanism of LOLA (Lunar Obiter Laser Altimeter) can avoid such error at least in the middle track. However, in the MOLA data analysis, it results in the erroneous local roughness crossing tracks. The capability to produce high resolution stereo DTMs is extremely useful to suppress the across track artefacts. For example, the maximum possible resolution (30-50m in our experiences) HRSC stereo DTMs are more effective than the gridded MOLA DTM to reduce across track artefacts. The CTX DTM covering $12-20 \mathrm{~m}$ spatial resolution is the optimal solutions. However, it is very difficult to find the sufficient enough CTX stereo coverage to rectify local roughness from MOLA beam broadening data. Moreover the geodetic control of CTX DTMs to be co-aligned with MOLA spots altogether is extremely difficult technical issue.

The practical solutions to suppress the across track artefacts of MOLA pulse width is the filtering in the spatial domain. In that cases, it is difficult to preserve the point density of along track points due to significance of the across track error.

Owing to such difficulties, the local roughness extracted from MOLA beam broadening effects are rarely employed for the martian topography research even with a few studies to construct local roughness map over Mars as Neumann et al., (2003) did. One exception can be founded in
Heavens et al., (2008)'s work to interpret martian boundary layer properties using the local roughness.

\subsection{Multi angle approaches}

A well known multi angle approaches for the local roughness extraction is the establishment of Hapke reflectance function and the inversion of roughness parameters from the fitted model (Hapke, 1993). It has been usually employed for the investigation of microscopic properties of regolith. Its effectiveness for the large scale geomorphic feature analysis was also demonstrated in Wu et al., (2008)'s work over terrestrial dune fields. However, the multiple optical imaging for Hapke modelling over target martian surface is not usually feasible. Hence, the simplified methods with multi angle imaging are required.

Two alternative approaches were proposed for this purpose. The first is the two-look surface roughness extraction suggested by Muskin and Gillespie (2005). The method is the simple division of the coregistrated pixel radiances in two look optical channels. Then the ratio of two pixels is

$$
\frac{R_{\text {sensor } 1}}{R_{\text {sensor } 2}}=\frac{\frac{1}{\pi} I_{1} \tau_{\text {sun } 1} \tau_{\text {sensor } 1} \rho_{1}\left(1-f_{\text {shadow } 1}\right)}{\frac{1}{\pi} I_{2} \tau_{\text {sun } 2} \tau_{\text {sensor } 2} \rho_{2}\left(1-f_{\text {shadow } 2}\right)}
$$

where $R_{\text {sensor } 1,2}$ is the measured radiance of multi angle channel 1 and 2, I is solar irradiance at top of atmosphere, $\tau_{\text {sun }}, \tau_{\text {sensor }}$ are atmospheric transmissivity in the sun and sensor surface paths, $\rho$ is the surface reflectivity and $\mathrm{f}_{\text {shadow }}$ is the effective fraction of the pixel area that is in shadow. In here the all terms except $f_{\text {shadow }}$ are canceled out or remain constant across the scene as long as the pixels in channels are correctly registrated. Then the radiance ratio depends on the surface roughness. After examining the idea with ASTER (Advanced Spaceborne Thermal Emission and Reflection Radiometer) over terrestrial surface, they applied the method for the THEMIS (Thermal Emission Imaging System) two look images and HRSC stereo channels in S1 and S2 (Muskin and Gillespie, 2006). Then it was verified compared with the in-suit measurements values and the thermal inertia map from THEMIS (Fergason et al., 2006)

The similar concept so called Normalised difference Angular Index (NDAI) to extract local roughness from multi angle observation was introduced by Nolin et al., (2002) for MISR (Multi-angle Imaging Spectro Radiometer) image.

$$
N D A I=\frac{R_{\text {forward }}-R_{\text {backward }}}{R_{\text {forward }}+R_{\text {backward }}}
$$

where $R_{\text {forvard }}$,backward is the bidirectional reflectance on the top of atomoshpere of forward and backward channels which have up to 60 degree view angles in the case of MISR. In the similar way with the two look approach, NDAI retains the effects of non-shadow fraction of pixels. Nolin and Payne (2007) employed NDAI to measure the local roughness of sea ice and glacier. Later, Kim et al., (2011) showed MISR NDAI effectively classify the sandy desert dune fields and the vegetated topography. Considering the sandy desert dunes have less variation in the meter scale than the vegetation canopy, the multi angle normalised radiance difference clearly reflects the variation of topographic heights. Therefore, it is worthwhile to evaluate the NADI and/or radiance ratio together compared with the other local roughness expressions.

\subsection{High resolution stereo DTM extractions and its applications for height variation parameters}

In order to fully utilise the data derived from image systems carried on various planetary orbiters, the generalised algorithms of stereo image processing and photogrammetric martian DTM extraction have been developed by Kim and Muller (2009). Due to the successful "from medium to high" control strategy, stable horizontal and vertical photogrammetric accuracy of resultant DTM was achievable, even without directly measured ground control information. The merits of this approaches is the highly correct geodetic control of DTM products. 
They produced CTX and HiRISE DTMs in the samples areas in the different image acquisition conditions. Then the resulting topographic data were compared with orbital laser altimetry spots. The comparison results between laser altimeter height points and stereo DTMs demonstrated extremely good agreement except a few rough topographies. In general, the procedures employing such strategy are able to produce $12-24 \mathrm{~m}$ resolution DTMs with CTX stereo and $3.5-0.5 \mathrm{~m}$ DTMs with HiRISE stereo respectively. Horizontally their geodetic accuracies are within few pixels and vertically the minor error within maximum 10-20m exists. Considering the spatial resolution of MOLA beam broadening local roughness or the products from multi-angle approaches, it can be used for the validation and the calibration of the other results.

For such purposes, it is important to define the local roughness parameters from the stereo intersection points. Shepard et al., (2001) proposed parameters to represent the surface local roughness from the height points. The RMS (Root Mean Squared) slope which is most commonly employed for the characterisation of terrain is shown as below

$$
\text { RMSslope }=\sqrt{\left[\frac{1}{n-1} \sum_{i=1}^{n}\left(z\left(x_{i}\right)-z\left(x_{i}+L\right)\right)^{2}\right] / L}
$$

where $L$ is the lag step, $n$ is the number of height point, $z\left(x_{i}\right)$ is the surface height at the location $x_{i}$ and $z$ is the average height of the surface. It's a variation of slopes in specific terrain range but very sensitive to the local scale noise. An alternative way to measure local roughness is the autocorrelation length. At first, autocorrelation is defined as,

$$
\text { autocorrelation }=\sum_{i=0}^{n-L-1}\left(x_{i}-\bar{x}\right)\left(x_{i+L}-\bar{x}\right) / \sum_{i=0}^{n-L}\left(x_{i}-\bar{x}\right)^{2}
$$

The lag length at which the autocorrelation value is less than $1 / e$ of zero lag autocorrelation is defined as the autocorrelation length. A parameter to representing local roughness in the different way is Hurst exponent.

$$
\begin{gathered}
\gamma(L)=\frac{1}{2 n} \sum_{i=0}^{n}\left[z\left(x_{i}\right)-z\left(x_{i}+L\right)\right]^{2} \\
H=\log (\gamma) / \log (L)
\end{gathered}
$$

Where $\gamma(\mathrm{L})$ is the semivariogram and $H$ is the Hurst exponent. The Hurst exponent represents the self similarity of topographic variation rather than roughness itself so it should be used with the other local roughness parameters. In this study, we constructed the local roughness maps of these parameters by the stereo techniques of Kim and Muller (2009).

\section{PROCESSING RESULT AND ASSESSMENT}

Our study sites are in the Athabasca valles, Eberswaldea crater and partly Iani valles. Eberswalde crater is an impact crater located at $24.2^{\circ}$ $\mathrm{S}$ latitude and $326.5^{\circ} \mathrm{E}$ longitude in the southern highlands of Mars, near north of Holden crater. It has been proposed as a potential landing site for a future rover mission organized by Mars Science Laboratory because of its clear fluvial origin.

The Athabasca valles (center latitude and longitude is $8^{\circ} \mathrm{N}$ and $156^{\circ} \mathrm{E}$ respectively) was a candidate for the landing place for the Mars Exploration Rover in addition to the scientific interest, considering the safety of a future rover in the well-eroded and extremely flat geomorphology.

Iani valles (center latitude and longitude is $3^{\circ} \mathrm{N}$ and $340^{\circ} \mathrm{E}$ respectively) is an part of Circum Chryse fluvial channels and well known by its importance in martian fluvial activities

\subsection{Local roughness based on stereo processing results \&}

Over the central part of the Athabasca area and Eberswalde crater, four different resolution DTMs (50m HRSC stereo DTM, 18m CTX stereo DTM 2-3m HiRISE DTM for whole HiRISE stereo area, 70cm DTM over some selected area) were extracted.
The geodetic control quality and the spatial resolving power of DTMs are the key issues for the purposes of this study. In Figure 1 (a), the constructed stereo DTMs showed very detailed but blunder free topographic features. The geodetic quality is highly accurate as shown in Figure 1 (b).

Then the local roughness parameters described in section 2.3 are extracted from stereo intersection points. To reduce the dependence on the slope, the de-trending to remove the influence of larger scale topography was also introduced as Shepard et al., (2001) did. After all, 2D local roughness maps over 2 testing area in Athabasca valles and Eberswalde crater were created (Figure 2).

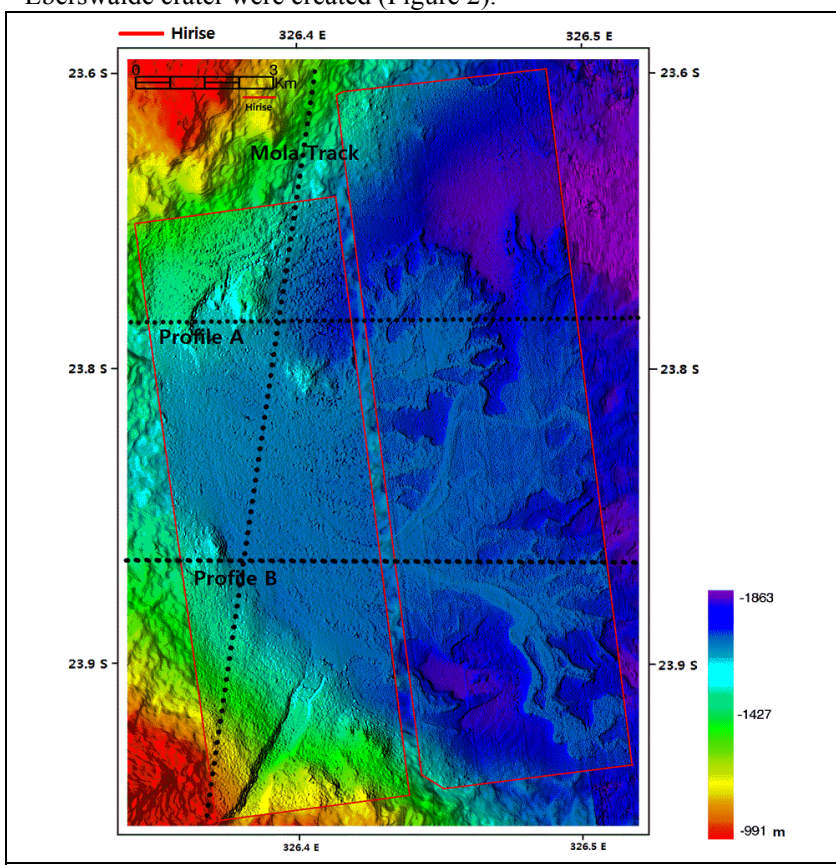

(a)Example of stereo DTM products over Eberswalde crater (20m CTX base DTM and two 2.5m HiRISE DTMs represented red box)

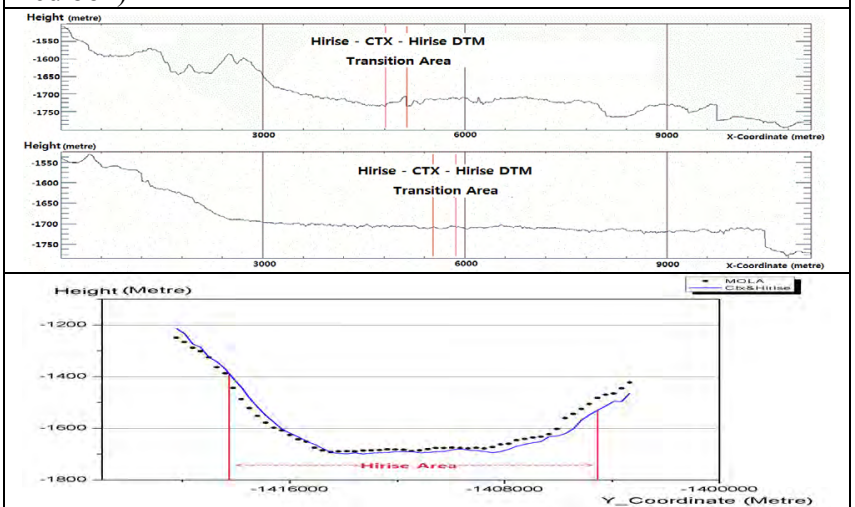

(b) geodetic quality check crossing profile over DTM mosaic using MOLA spot

Figure 1. Example of stereo DTM products over Eberswalde crater and geodetic quality check

In Figure 2, RMS slope and Hurst constant map demonstrate the similar topographic characteristics compared with the textures from optical image. Autocorrelation length is highly noisy but less correlated with the long baseline slope. Since the stereo intersection points from the 2$3 \mathrm{~m}$ resolution HiRISE image were used for such local roughness extraction, these local roughness maps do not directly represent the meter/sub meter local roughness. However, Hurst constant expressing the self similarity is highly correlated with RMS slope so that possibly the local roughness properties in this resolution could be inherited up to sub meter level. The full proof of this assumption will be shown in next stage research which will employ more sophisticated matching algorithm to produce sub meter resolution stereo intersection points. 
In here, the extracted parameters showed the potential to make quantification of surface local roughness from the direct measurements.
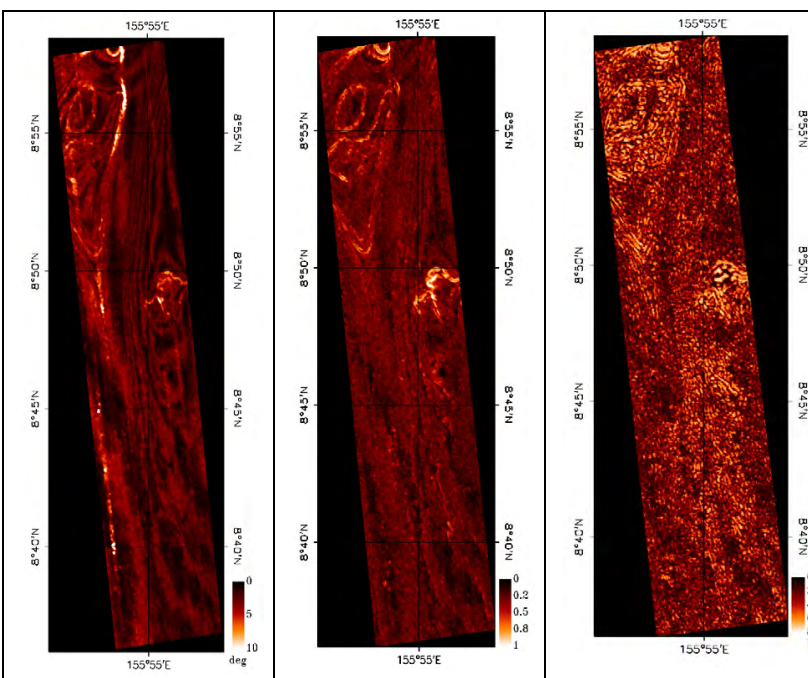

(a)local, roughness analyse over Athabasca valles
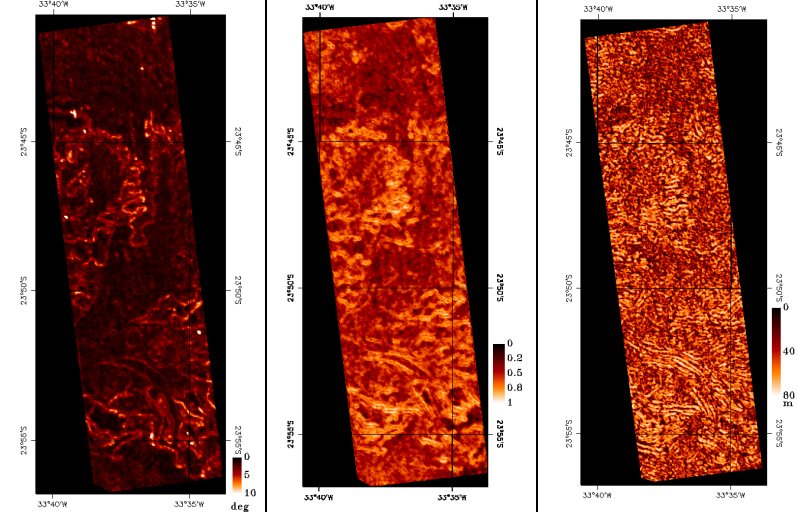

(b) local, roughness analyse over Eberswalde crater

Figure 2. Local roughness analyses (left : RMS slope, middle : Hurst constant, right : autocorrelation length)

\subsection{MOLA local roughness results}

The precondition of local roughness extraction from MOLA beam broadening is the effective suppression of across track drifts. First of all, the most reliable base DTMs using HRSC DTMs with DLR-VICAR photogrammetric routines (Scholten et al., 2005) and our image matching scheme were extracted. Then the noisy tracks are removed by the comparison between the altimetry height values and DTM. The correction result using 50m HRSC DTMs over Inai Valles is showing the better qualities compared with the output with MOLA height point. $500 \mathrm{~m}$ MOLA gridded DTM which is coarser than the footprint size of altimeter beam is inadequate to correctly rectify the slope effect in beam broadening as shown in Figure 3 (a).

It is desirable to employ CTX DTM $(<20 \mathrm{~m})$ to precisely rectify the slope effect. However, the sufficient CTX stereo coverage over target area is not available so that over a part of Eberswalde crater, the slope correction using $18 \mathrm{~m}$ CTX stereo DTM was applied. In addition, the adaptive filtering to remove artifacts crossing tracks in the pulse beam broadening was performed. The full across track drift removing is not possible yet so that it results in the low resolution roughness map as shown in Figure 3 (b). With all above methods, the maximum resolution not showing significant across track drift in roughness map is usually $1 / 32-1 / 64$ degree (about $1 \mathrm{~km}$ in equator). It is far lower that the MOLA spot density as well as currently available MOLA gridded DTM resolution.

After all, Figure 3 is demonstrating the constructed local roughness map over Iani valles and Eberswalde test areas. The correlations with the slope should result from the remnants of uncorrected slope effects in laser beam broadening as well as the self similarity of natural topographic.

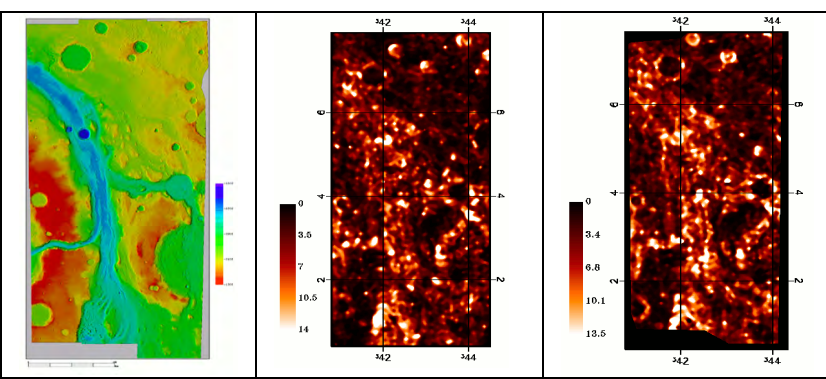

(a) The corrected local roughness map using MOLA gridded DTM (middle) and HRSC DTM over Iani Valley (right)
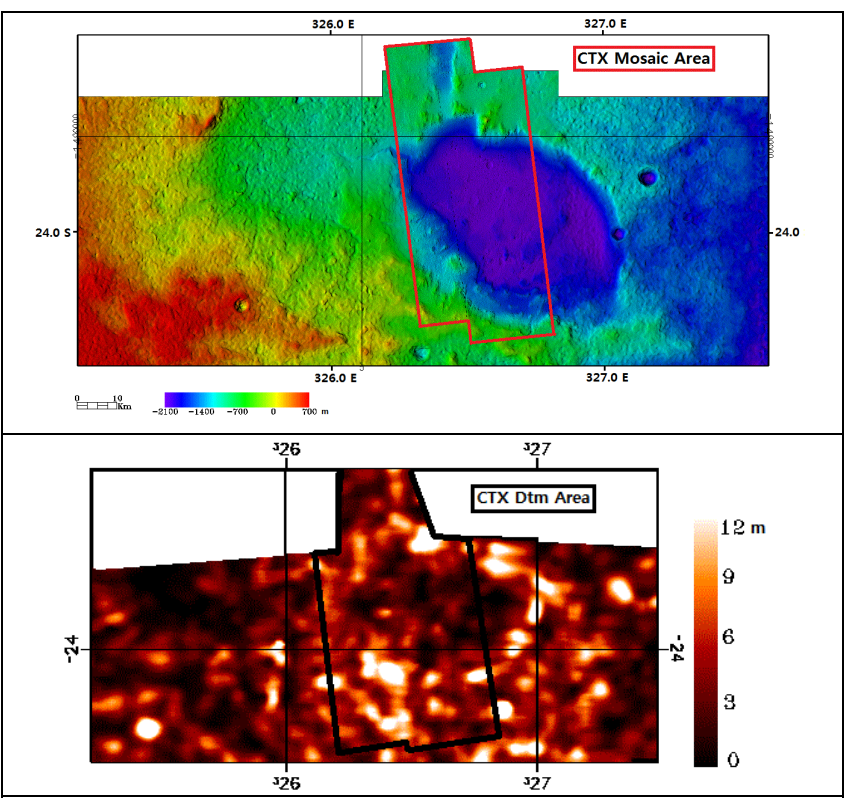

(b) The corrected local roughness map using HRSC and CTX DTM over Eberswalde

Figure 3. The corrected local roughness map based on the MOLA beam broadening effect

\subsection{Multi angle local roughness results}

The precondition of multi angle local roughness is the highly accurate pixel registration between the different viewing channels. The non georectified level 2 HRSC images employment as done by Muskin and Gillespie, (2006) is not suitable solution for the martian topography analysis. It can be satisfied by the level 4 ortho image creation using the high accuracy DTMs and then the extraction of multi angle parameters. Therefore, we firstly created 50m resolution HRSC DTMs over testing areas (see the detailed technical procedure in Kim and Muller (2009)). Due to the sub pixel matching capability of Gruen (1985)'s algorithms, the corresponding pixels in HRSC level $4 \mathrm{~S} 1$ and S2 channels were placed on the exactly the sample map coordinate. Compared with MISR case, the angle difference between $\mathrm{S} 1$ and $\mathrm{S} 2$ channel is relatively small so that the local roughness from this analysis was not well segregated from the slope effect. The testing results with pixel ratio and NDAI method didn't show big difference so NDAI was used for local roughness analysis in this study. Figure 4 demonstrated the extracted NDAI over target areas. In spite of the seriously impaired products by the illumination condition and the image noise, the roughness distributions in the flat areas were clearly shown. 


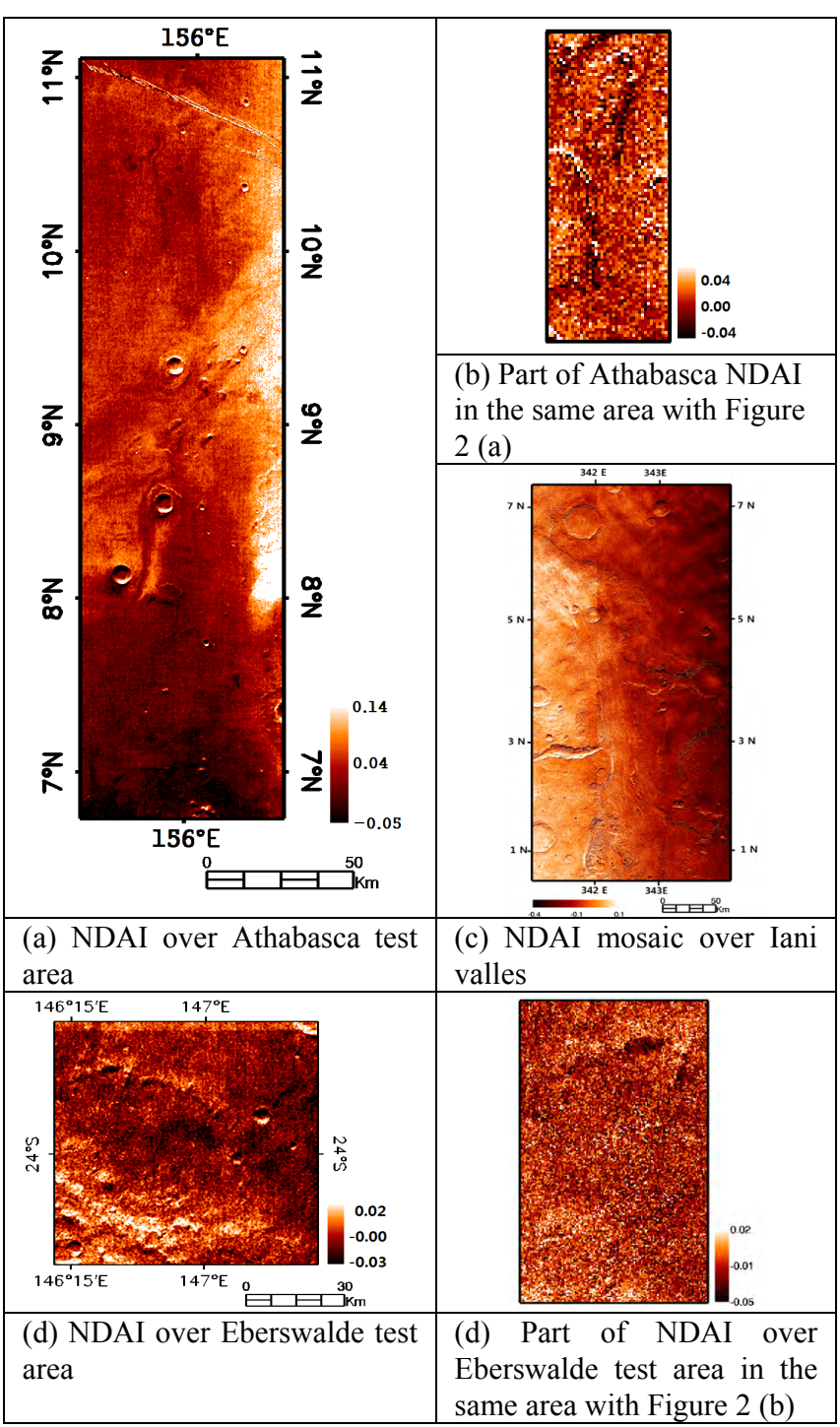

Figure 4. NDAI local roughness analysis by multi angle imaging

\subsection{Calibration and comparison}

The extracted results from the beam broadening approach and the multi angle analysis are not calibrated for the quantification of topographic roughness. Currently, the mathematical modelling of the beam broadening approach and the multi angle parameters with the topographic height variation are not available. To convert them to the absolute quantization of the height variation, the cross verification between the local roughness based on stereo analysis and the other results is necessary. It requires highly accurate registration between the HiRISE, CTX, HRSC stereo products and MOLA data sets which were employed to extract the local roughness parameters. The condition was satisfied by the coarse to fine geodetic control strategy (see Kim and Muller (2009) and Kim et al., (2007) about the technical aspect of the geodetic control). Figure 5 showed the comparison results of the beam broadening and multi look parameter using the RMS slope values from the stereo intersection points. In the case of multi angle parameter approach, the correlation with RMS slope was very weak due to the HRSC image noise in the test areas as shown in Figure 5 (b). It should be noted the investigation of such correlation relationship were performed only two test areas. Therefore, the conversion given (9) in is very rough and initial. After manually removing the outliners, the relationship is given as below

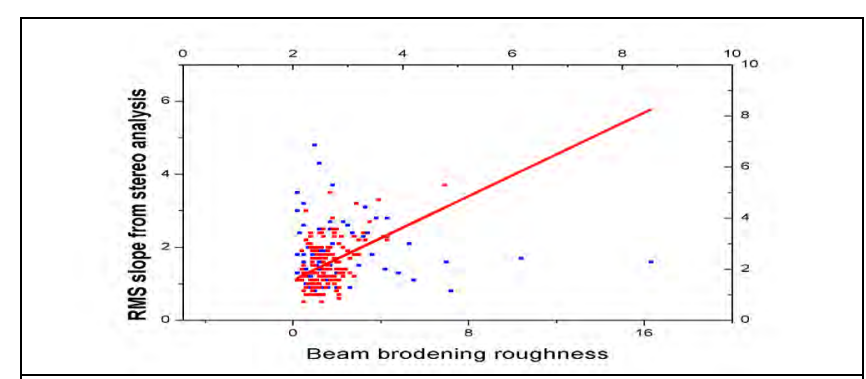

(a) The compassion between MOLA beam broadening and stereo analysis (blue : Eberswalde observation points, red : Athabasca observation points)

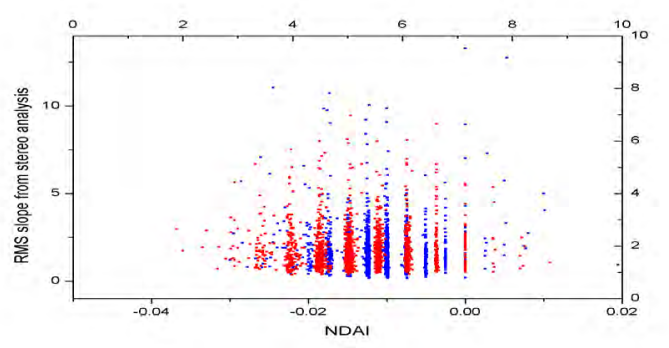

(b) The compassion between multi angle analysis and stereo analysis (blue : Eberswalde observation points, red : Athabasca observation points)

Figure 5. The correlation between the local roughness parameter by stereo analysis and the other results

\section{CONCLUSIONS \& FUTURE WORK}

To extract the local roughness parameters from the planetary topography, three methods were tested here. The laser beam broadening effect analysis with the full coverage over martian surface still showed unresolved across track artifacts even with the slope correction by the best possible DTM source and the adaptive filtering. The multi angle approaches were suffered by the strong correlation with the topographic slope and the noises in the flat surfaces. The direct extractions of Hurst constant, RMS slope and autocorrelation length from height points demonstrate similar distributions each other. The correlation between the slope and the local roughness parameters from the laser beam broadening approach and the multi angle analysis may result from the self similarity of target topography and the uncorrected slope terms.

Since all data sources employed in this study are well co-registered, it is possible to compare the extracted local roughness parameters with the direct measurement from 2-3 meter resolution HiRISE stereo height points. As the result, the roughness by multi angle approach and the parameters by the stereo analysis showed very weak correlation probably by the image noise. The calibration of the beam broadening by the stereo height point analysis was tried and produced the conversion parameters. However, it should be noted that two sample areas for the calibration are not enough to estimate stable conversion parameters. The introduction of more stereo analysis results is required for the correct calibration of the local roughness parameters from medium resolution data sets.

As demonstrated in Marticorena et al., (2006) work, if the roughness parameters are converted into the aerodynamic roughness length Zo with the help of wind tunnel experiment, it will be extremely useful to classify planetary surfaces by their origins and provide important clues to simulate the surface process over target planet. Together with it, the detailed modeling of beam broadening effect and multi look parameters with the parameters extracted from the fine resolution data analysis such as sub meter HiRISE stereo intersection points are strongly required.

Since the local roughness data is also powerful means to assess the risk of potential landing sites with quantitative engineering standard, we will performed our local roughness processing strategy over potential MSL(Mars Science Laboratory) landing sites. 
Bagnolds, R. A., 1941. The Physics of Blown Sand and Desert Dunes London: Methuen

Fergason, R. L., P. R. Christensen, J. F. Bell III, M. P. Golombek, K. E. Herkenhoff, and H. H. Kieffer, 2006. Physical properties of the Mars Exploration Rover landing sites as inferred from Mini-TES-derived thermal inertia, Journal of Geophysical Research, 111, E02S21, doi: $10.1029 / 2005$ JE002583

Gardner, C.S., 1992. ranging performance of satellite laser altimeters, IEEE Transaction on Geoscience and Remote Sensing, 30 (5), pp.1061-1072,

Gruen, A. 1985. Adaptive Least Squares Correlation: A powerful Image Matching Technique. South Africa Journal of Photogrammetry, Remote Sensing and Cartography.14(3), pp. 175-187.

Golombek, et al., 2003. Selection of the Mars Exploration Rover landing sites. Journal of Geophysical Research, 111(E12), doi:10.1029/2003JE002074

Hapke, B.W., 1993. Theory of reflectance and Emittance Spectroscopy. Cambridge Univ. Press, New York.

Heavens, N.G., Richardson, M.I., and Toigo, A.D., 2008. Two aerodynamic roughness maps derived from Mars Orbiter Laser Altimeter (MOLA) data and their effects on boundary layer properties in a Mars general circulation model (GCM), Journal of Geophysical Research, 113, E02014, doi:10.1029/2007JE002991

Kim J-R., et al., 2007. Geometric Ground Control of Very High Resolution Imagery Using HRSC Intersection Points and a Non-Rigorous Camera Model, LPSC 2007, 12-14, March,2007, Houston, Tx, USA. http://www.lpi.usra.edu/meetings/lpsc2007/pdf/1811.pdf

Kim, J.-R. and Muller, J.-P., 2008. Very high resolution stereo DTM extraction and its application to surface roughness estimation over Martian surface. International Archives of the Photogrammetry, Remote Sensing and Spatial Information Sciences, 37(B4): pp.993-998.

Kim, J. R., and Muller, J-P., 2009. Multi-resolution topographic data extraction from Martian stereo imagery, Planetary and Space Science, 57 (15),pp.2095-2112.

Kim, J. R., Yun, J., Choi, Y.S. and Yun, H.W., 2011. The correlation analysis between the dust storm in Far East Asia and the dynamic local roughness in northwest China sandy desert by remote sensed data,
European Goescience Union 2011

Neumann, G. A., Abshire, J. B., Aharonson, O., Garvin, J. B., Sun, X., and Zuber, M. T., 2003. Mars Orbiter laser Altimeter pulse width measurements and footprint-scale roughness, Geophysical Research Letter, 30(11), 1561

Nolin, A. W., Fetterer, F. M., and Scambos, T. A. 2002. Surface roughness characterizations of sea ice and ice sheets: Case studies with MISR data. IEEE Transaction on Geoscience and Remote Sensing, 40(7), pp.1605-1615.

Nolin. A. W., and Payne, C. M., 2007. Classification of glacier zones in western Greenland using albedo and surface roughness from the Multi-angle Imaging SpectroRadiometer (MISR), Remote Sensing of Environment ,107, pp.264-275

Marticorena, B., and Bergametti, G., 1995. Modeling the atmospheric dust cycle: 1. Design of a soil derived dust production scheme, Journal of Geophysical Research, 100, 16, pp.415-16,430.

Marticorena, B., et al., 2006. Surface and aerodynamic roughness in arid and semiarid areas and their relation to radar backscatter coefficient, Journal of Geophysical Research, 111, F03017, doi:10.1029/2006JF000462.

Mushkin, A., and Gillespie, A. R., 2005. Estimating sub-pixel surface roughness using remotely sensed stereoscopic data, Remote Sensing of Environment., 99, pp.75-83.

Mushkin, A., and Gillespie, A. R., 2006. Mapping sub-pixel surface roughness on Mars using high-resolution satellite image data, Geophysical Research Letter, VOL. 33, L18204.

Scholten, F., Gwinner, K., Roatsch, T., Matz, K.D., Wählisch, M., Giese, B., Oberst, J., Jaumann, R., Neukum, G., 2005. Mars Express HRSC data processing - Methods and operational aspects. Photogrammetric Engineering \& Remote Sensing, 71(10), pp.1143-1152.

Shepard, M. K., Campbell, B. A., Bulmer, M.K., Farr, T.G., Gaddis, L.R., Plaut, J.J., 2001. The Roughness of Natural Terrain : A Planetary and Remote Sensing Perspective, Journal of Geophysical Research, 106(E12), pp.32777-32795.

Wu, Y., Gong, P., Liu, Q. and Chappell, A. 2009. Retrieving photometric properties of desert surfaces in China using the Hapke model and MISR data. Remote Sensing of Environment, 113, 1, pp. 213-223. 